\title{
Conoscopic holography: two-dimensional numerical reconstructions
}

\author{
Laurent M. Mugnier \\ Télécom Paris, Département Images, 46 rue Barrault, F-75634 Paris Cedex 13, France \\ Gabriel Y. Sirat and Didier Charlot \\ Le Conoscope S.A., 12 Avenue des Prés, F-78180 Montigny Le Bretonneux, France
}

Received June 29, 1992

\begin{abstract}
Conoscopic holography is an incoherent light holographic technique based on the properties of crystal optics. We present experimental results of the numerical reconstruction of a two-dimensional object from its conoscopic hologram.
\end{abstract}

Conoscopic holography $(\mathrm{CH})$ is a spatially incoherent light holographic technique ${ }^{1-3}$ developed primarily as a three-dimensional imaging and measuring technique. Several systems are in development today; they include a range finder, ${ }^{4}$ a profilometer, and a microscope.

We have seen the reconstruction of two-dimensional objects as an important and necessary step, with the aim of characterizing and quantifying the performances of this technique. Indeed, it seems unreasonable to try to reconstruct three-dimensional objects before obtaining good two-dimensional image reconstructions. It is the study of such two-dimensional reconstructions that is presented in this Letter.

As with other similar techniques ${ }^{5,6}$ each object point produces, on the recording plane, a Gabor zone pattern (GZP) that encodes both its lateral and longitudinal positions, and the hologram is the incoherent superposition of such GZP's. In $\mathrm{CH}$, these patterns are formed in the following way: a uniaxial crystal is sandwiched between two circular polarizers (Fig. 1); in the crystal, the monochromatic wave from each point is split equally into two (ordinary and extraordinary) waves, traveling with different velocities. These waves are recombined by the output polarizer, which converts the phase difference into an amplitude modulation. The hologram is recorded on a CCD camera rather than on a photographic plate so as to enable its numerical processing.

When the object is a single monochromatic point and when the crystal axis is parallel to the geometrical axis $O z$ of the system, the hologram is the point-spread function (PSF), $R_{c}^{+}$, which is a bias plus the above-mentioned real GZP ${ }^{1-3}$ :

$$
\begin{aligned}
R_{c}^{+}(x, y)= & \frac{1}{2}\left\{1+\cos \left[\pi f_{r}\left(x^{2}+y^{2}\right)\right]\right\} \\
= & \frac{1}{2}+\frac{1}{4} \exp \left[-i \pi f_{r}\left(x^{2}+y^{2}\right)\right] \\
& +\frac{1}{4} \exp \left[i \pi f_{r}\left(x^{2}+y^{2}\right)\right],
\end{aligned}
$$

where $x$ and $y$ are the coordinates in the recording plane and $f_{r}$ is a scale factor that we call the Fresnel parameter, which depends on the distance between the point and the recording plane. ${ }^{3}$ The first term in Eq. (1) is the so-called bias, which is an important problem in incoherent holography ${ }^{6,7}$ and the second term gives the conjugate image in the reconstruction.

In Ref. 8 we recalled how to modify the system in order to remove the bias and addressed the removal of the conjugate image. These improvements are based on numerically combining different system PSF's, each of which is obtained by adequately changing the input polarization state [with a liquid-crystal light valve (LCLV)] and modulating the amplitude (with a rotating mask) of the incident light field (see Ref. 8 and Fig. 2). The resulting PSF is shown to be

$$
R_{e}(x, y)=\exp \left[i \pi f_{r}\left(x^{2}+y^{2}\right)\right] .
$$

$R_{e}$ is the sole third term of Eq. (1) and consequently will give neither bias nor conjugate image in the reconstruction. If the object is planar, which is the case that we address here, $f_{r}$ is constant, so that the relationship between the image $I$ of the object and hologram $H$ is a two-dimensional convolution

$$
H=I \otimes R_{e} .
$$

The reconstruction, which consists of recovering the image $I(x, y)$ of the object (and, possibly, its distance to the recording plane), is a (linear) deconvolution problem. The transfer function of the system

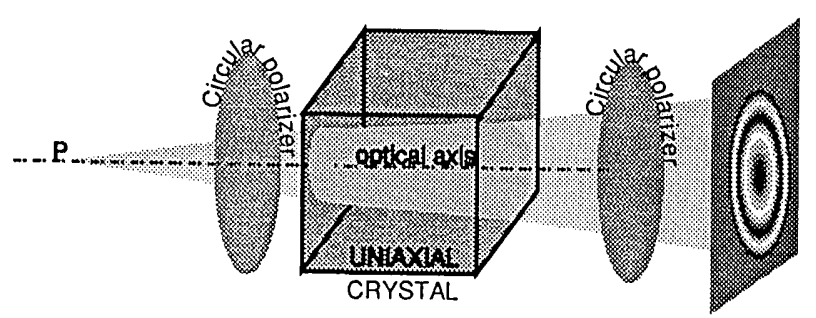

Fig. 1. Basic experimental setup. A uniaxial crystal is sandwiched between two circular polarizers. When a point source $P$ illuminates the system, a GZP is observed at the output. 


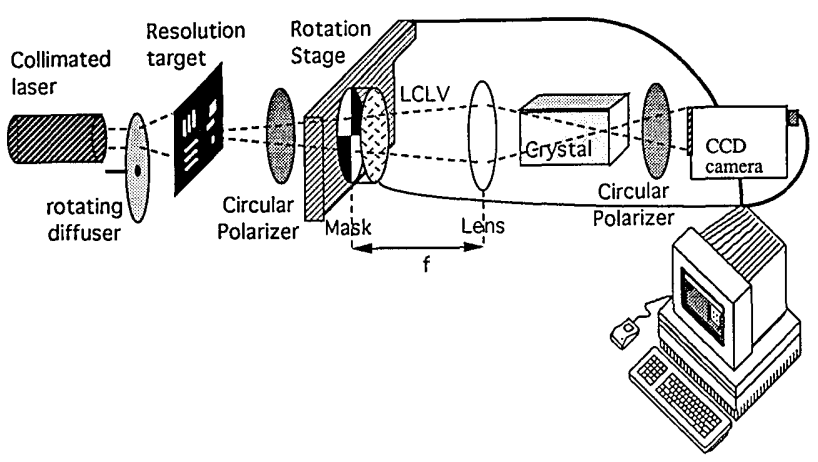

Fig. 2. Experimental setup for the acquisition of twodimensional objects.

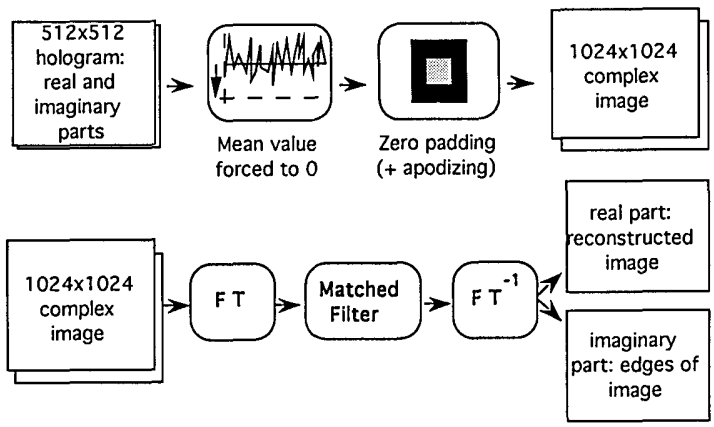

Fig. 3. Numerical processing of the recorded hologram. FT, Fourier transform.

is given by

$$
\tilde{R}_{e}(\mu, \nu)=\frac{i}{f_{r}} \exp \left[\frac{-i \pi}{f_{r}}\left(\mu^{2}+\nu^{2}\right)\right] .
$$

This transfer function has a constant modulus so that the inverse filter is, within a constant factor, equal to the matched filter (by definition the transfer function of the matched filter is the conjugate of the transfer function of the system). Because $R_{e}$ is even, the matched filter is also, in the spatial domain, the conjugate of $R_{e}$. The numerical reconstruction of the image $I$ consequently appears to be the simulation of the backpropagation of a coherent light wave from the hologram to the object plane and reads

$$
H \otimes R_{e}^{*}=I \otimes R_{e} \otimes R_{e}^{*}=I \otimes \frac{1}{f_{r}^{2}} \delta=\frac{1}{f_{r}^{2}} I .
$$

Theoretically, the imaginary part of the reconstruction is zero because the image $I$ is real. In practice, the imaginary part of the reconstruction is minimum when the Fresnel parameter $f_{r}^{\prime}$ of the reconstruction kernel $R_{e}^{\prime}$ is equal to that of the acquisition (i.e., when $R_{e}{ }^{\prime}$ is equal to $R_{e}{ }^{*}$ ) but is not zero. For $f_{r}^{\prime}$ close to $f_{r}$, which corresponds to a slight defocus of the reconstruction, a first-order Taylor development of the transfer function of the whole process (acquisition plus reconstruction) is

$$
\tilde{R}_{e} \cdot \tilde{R}_{e}{ }^{\prime} \approx \frac{1}{f_{r}^{2}}\left[1+\frac{i \pi\left(f_{r}-f_{r}^{\prime}\right)}{f_{r}^{2}}\left(\mu^{2}+\nu^{2}\right)\right] .
$$

In the spatial domain, the reconstructed image is then

$$
\begin{aligned}
H^{\prime}(x, y) & =H \otimes R_{e}{ }^{\prime}(x, y) \\
& =\frac{1}{f_{r}^{2}} I(x, y)+\frac{i\left(f_{r}^{\prime}-f_{r}\right)}{4 \pi f_{r}^{4}} \Delta I(x, y),
\end{aligned}
$$

where $\Delta=\partial^{2} / \partial x^{2}+\partial^{2} / \partial y^{2}$ denotes the Laplacian operator. The imaginary part of the reconstruction thus contains the edges of the original object for any nonzero defocus.

A useful parameter for the numerical processing of GZP's is the number of black and white fringes $F$ recorded on a given sensor; if the sensor is of halfwidth $R$ then $F$ is related to the Fresnel parameter of Eq. (1) by

$$
F=f_{r} R^{2} .
$$

It has been shown ${ }^{2}$ that the theoretical maximal resolution of the reconstructed image is equal to the resolution of the hologram itself (that is to say, the resolution of the CCD sensor) and is achieved when the hologram is sampled at the Nyquist rate. The correct sampling condition, for a sensor having

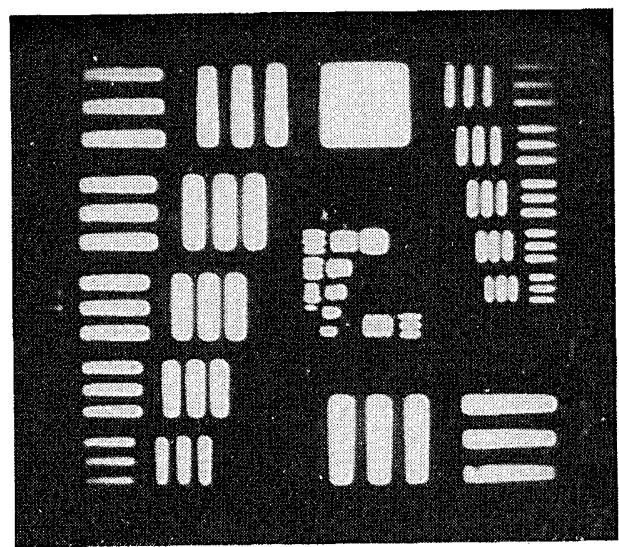

Fig. 4. Object (three-bar resolution target) as seen directly by the CCD camera.

(a)

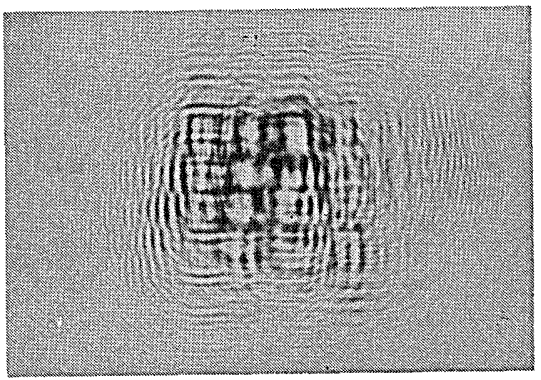

(b)

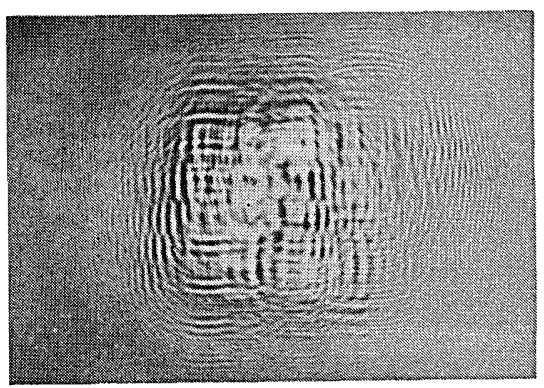

Fig. 5. (a) Real and (b) imaginary parts of the recorded hologram. 
(a)

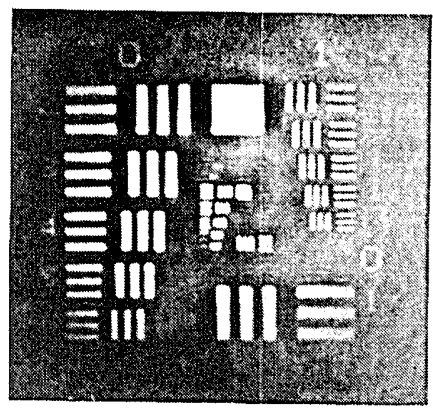

(b)

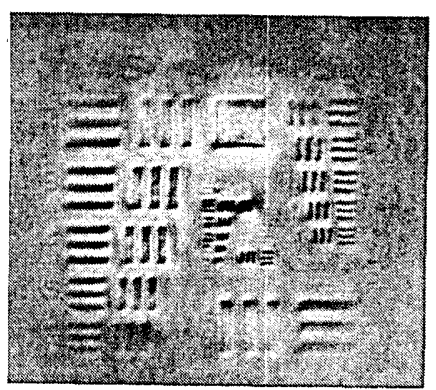

Fig. 6. (a) Real and (b) imaginary parts of the numerical reconstruction.

$N$ pixels, takes the simple form

$$
F \leq N / 4 \text {. }
$$

In our setup, $N$ equals 512 so that the optimum number of fringes $F$ is 128 . For $F$ smaller than this optimum, the resolution (in number of points) of the reconstructed image will be lower; more precisely, the size of a reconstructed point will be the width of the outermost recorded fringe.

We have recorded the conoscopic hologram of a resolution target on a setup (Fig. 2) consisting of the following: (1) a collimated 10-mW He-Ne laser that is used for alignment, calibration (acquisition of the PSF), and the acquisition of objects; (2) a three-bar resolution target as object, with a rotating ground-glass diffuser placed before it to eliminate speckle; (3) the mask (gray-level slide transferred onto a photographic plate) and the PC-driven LCLV (Meadowlark LVR-0.7-CUS), mounted together on a rotation stage (Microcontrôle, also PC driven); (4) a $50-\mathrm{mm} f / 1.8$ Nikkor lens with the mask in its front focal plane to image the object into the system; and (5) a 50-mm-long calcite crystal $(\phi=20 \mathrm{~mm})$, the output circular polarizer, and a CCD camera (Cohu 4712) whose images are digitized on $512 \times 512$ pixels (Matrox board).

The different steps of the digital processing that constitutes the reconstruction are described in Fig. 3. This numerical reconstruction is essentially, as shown above, the simulation of a coherent optical reconstruction. Figure 4 shows the target as seen directly by the CCD camera (when the crystal, the mask, and the LCLV are removed). Figures 5(a) and 5(b) show the real and imaginary parts of the recorded hologram.

The LCLV delay strongly depends on temperature, which accounts for the following imperfection of the PSF: a small leftover bias is present in the imaginary part of the hologram and would give a visible
Fresnel diffraction pattern at the edges of the reconstructed image if we did not numerically subtract its mean value from the hologram. Figures 6(a) and 6 (b) show the real and imaginary parts of the numerical reconstruction, which are free from the conjugate image. Notice that the few dead pixels that are visible on the lower large horizontal bar of the image (Fig. 4) are no longer visible on the reconstruction [Fig. 6(a)], which is due to the information multiplexing feature of the hologram. The number of fringes, measured by replacing the target with a centered point source, is $F=112$. For any noncentered point, the number of visible fringes on the sensor will be greater than this and close to the optimum, i.e., 128. In the horizontal direction, the comparison between original and reconstructed images shows that we have reached the theoretical resolution. The resolution is not so good as in the vertical direction because the CCD sensor is rectangular (and smaller in the vertical direction). The slight diffraction pattern, consisting of horizontal fringes superimposed on the reconstructed image, is also an effect of the truncation of the hologram in the vertical direction. This defect can be suppressed without significantly decreasing the resolution by apodizing the hologram with an appropriate window. ${ }^{9}$

The main goal of the study presented in this Letter was to characterize $\mathrm{CH}$ experimentally. However, in the course of this work, several applications of this technique to two-dimensional objects emerged. Indeed, by using $\mathrm{CH}$, it is possible to build a twodimensional imaging system in which the final focusing step is done after the recording of the data. The main application of such a system would be for imaging under unstable conditions, i.e., with a rapidly changing focusing distance. A second possible application is to image objects consisting of several planes (for example, in microelectronics).

In conclusion, we have presented an experimental reconstruction from a conoscopic hologram of a (planar) resolution target that has no conjugate image and reaches the theoretical resolution limit. The quality of the reconstructed image will allow us to present reconstructions of three-dimensional objects in the near future.

Gabriel Y. Sirat is on leave from Télécom Paris. Didier Charlot was the technical director of Le Conoscope S.A.

\section{References}

1. G. Y. Sirat and D. Psaltis, Opt. Lett. 10, 4 (1985).

2. D. Charlot, "Holographie conoscopique, principe et reconstructions numériques," Ph.D. dissertation (Ecole Nationale Supérieure des Télécommunications, Paris, 1987).

3. G. Y. Sirat, J. Opt. Soc. Am. A 9, 70, 84 (1992).

4. D. Charlot, L. M. Mugnier, and G. Y. Sirat, Proc. Soc. Photo-Opt. Instrum. Eng. 1265, 52 (1990).

5. A. W. Lohmann, J. Opt. Soc. Am. 55, 1555 (1965).

6. G. Cochran, J. Opt. Soc. Am. 56, 1513 (1966).

7. A. Kozma and N. Massey, Appl. Opt. 8, 393 (1969).

8. L. M. Mugnier and G. Y. Sirat, Opt. Lett. 17, 294 (1992),

9. F. J. Harris, Proc. IEEE 66, 51 (1978). 